\title{
A propos d'un accident survenu à une conduite forcée après quarante ans d'exploitation
}

\section{A 40-year-old penstock bursts}

\author{
PAR (x. FERRAND
}

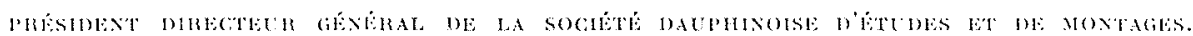

\begin{abstract}
Rupture de la conduite forcée de Champagny installée en 1912. Par contre-coup, la conduite récente voisine de Pralognan subit, sans dommages sérieux, une épreube toul à fait exceptionnelle. Les lecons de cet accident : insuffisance des marges de sécurité almises il y a quarante ans; justification des règles imposées en France; intéret incomparable des nouneaux procédés de fabrication qui constituent une nérification expérimentale certaine de la résislance rélle du tuyaut.
\end{abstract}

\begin{abstract}
Bursting of the Chompronny penstock, installed in 1912. The neably Iralogman penstock. recently installed, undergoes an unexpected and exceptionat lest without any serions damuge. The lessons tearnt from this accident: insufficiency of the safely margins alloumed to years rgo-iustifications of the rutes imposed in France incomparable adeantages of the new manufacturing methods which form an whquestionable experimental verification of the trae resistance of the pipe
\end{abstract}

Le 15 novembre 1952, une rupture s'est produite sur un tuyau de la conduite forcée dite du "Doron de Champangy », alimentant les turbines de l'usine de Bozel-Malétra.

Celte conduite, construite en 1912, fonctionno sous une chute brute de $550 \mathrm{~m}$ environ, sa longueur totale est de $1.300 \mathrm{~m}$ environ, divisée $\mathrm{cn}$ trois diamètres de 950 , 850 et $800 \mathrm{~mm}$ (fig. 1).

La partic supérieure, sur $300 \mathrm{~m}$ environ, esl

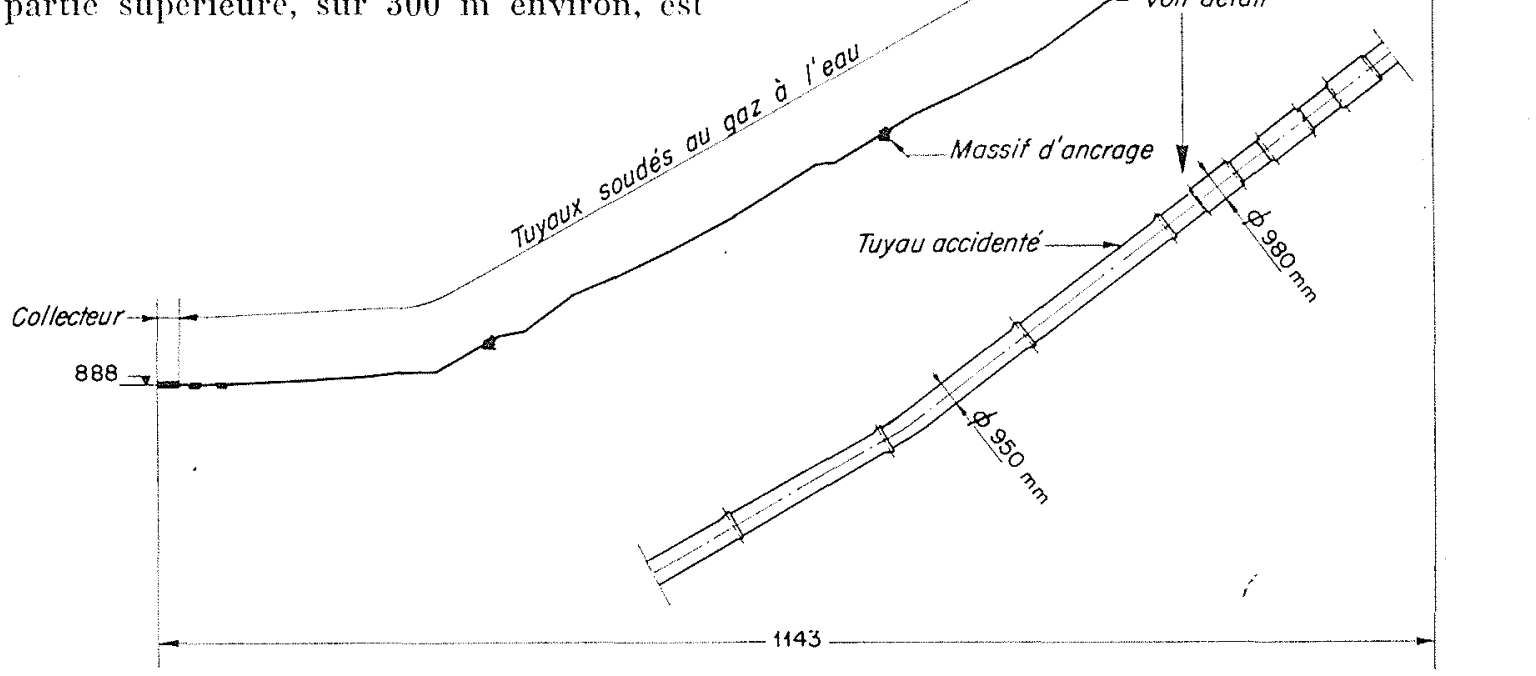

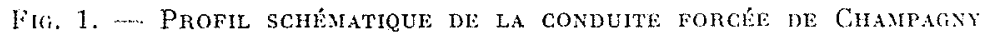
DT DE LA PORTION ACCIDENTÉE. 


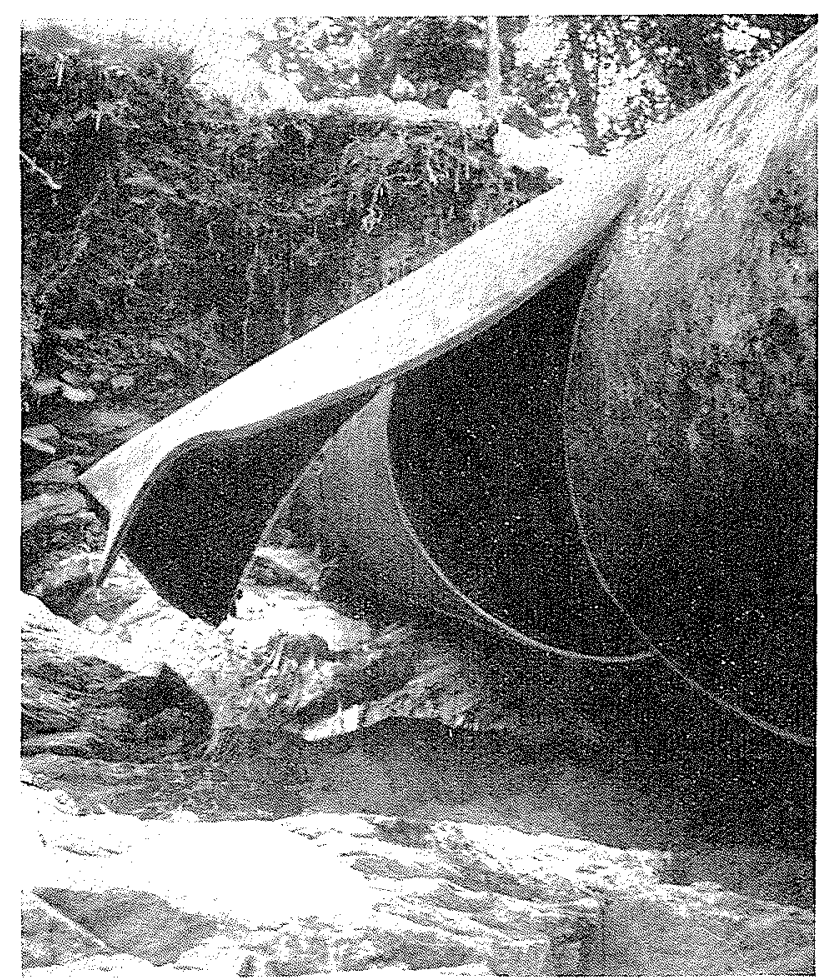

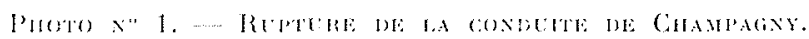

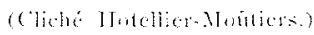

normal de marche, donne une pression de calcul de $195 \mathrm{~m}$ environ et une fatigue en pleine tole de $8 \mathrm{~kg} / \mathrm{mm}^{2}$ environ.

La rupture semble s'ètre anorcée sur une soudure longitudinale en partant de l'amont, it la rivure d'emboitement avec les tuyaux rivés. La conduite s'est ouverte sur $1,600 \mathrm{~m}$ de longueur environ, la cassure est nette et rectiligne, sans défauts apparents sur les tranches de la cassure.

Il est à noter que les tuyaux de cette conduite étaient, à l'époque de sa construction, éprouvés en usine à 1,5 fois la pression de marche, alors que depuis 1918 tous les tuyaux sont éprouves at 2 fois la pression maximum de marche $\mathrm{P}$, condition considérée comme nécessaire et suffisante pour qu'en exploitation aucune rupture conséquente à une surpression exeeptionnelle et aceidentelle ne se produise. Il est certain que si un tel tuyau avait subi une telle épreuve, la rupture se serait produite en usine et non sur place, entre 1,5 et $2 \mathrm{P}$.

La vanne de sécurité en lète de la conduile n'a pas fonctionné et l'eau s'est écoulée à l'extérieur pendant deux heures, le long des deux nouvelles eonduites de la chute dite de « Pralognan », installées à proximité en 1947-1948 of comprenant une conduite de décharge et une conduite forcée (fig. 2, photo n" 2). Ces condui-

construite en luyaux rivés de 6 a 15 mon d'épaisseur et le reste en tuyaux soudés au gat ì l'eau de 12 à $30 \mathrm{~mm}$.

La rupture s'est produite sur le luyau soudé alu gaz à l'eau se raccordant avec les tuyaux rivés (photo $n^{\circ} 1$ ). La pression statique à cet cndroit est de $175 \mathrm{~m}$ environ, ce qui, avec la surpression due all coup de bélicr maximum

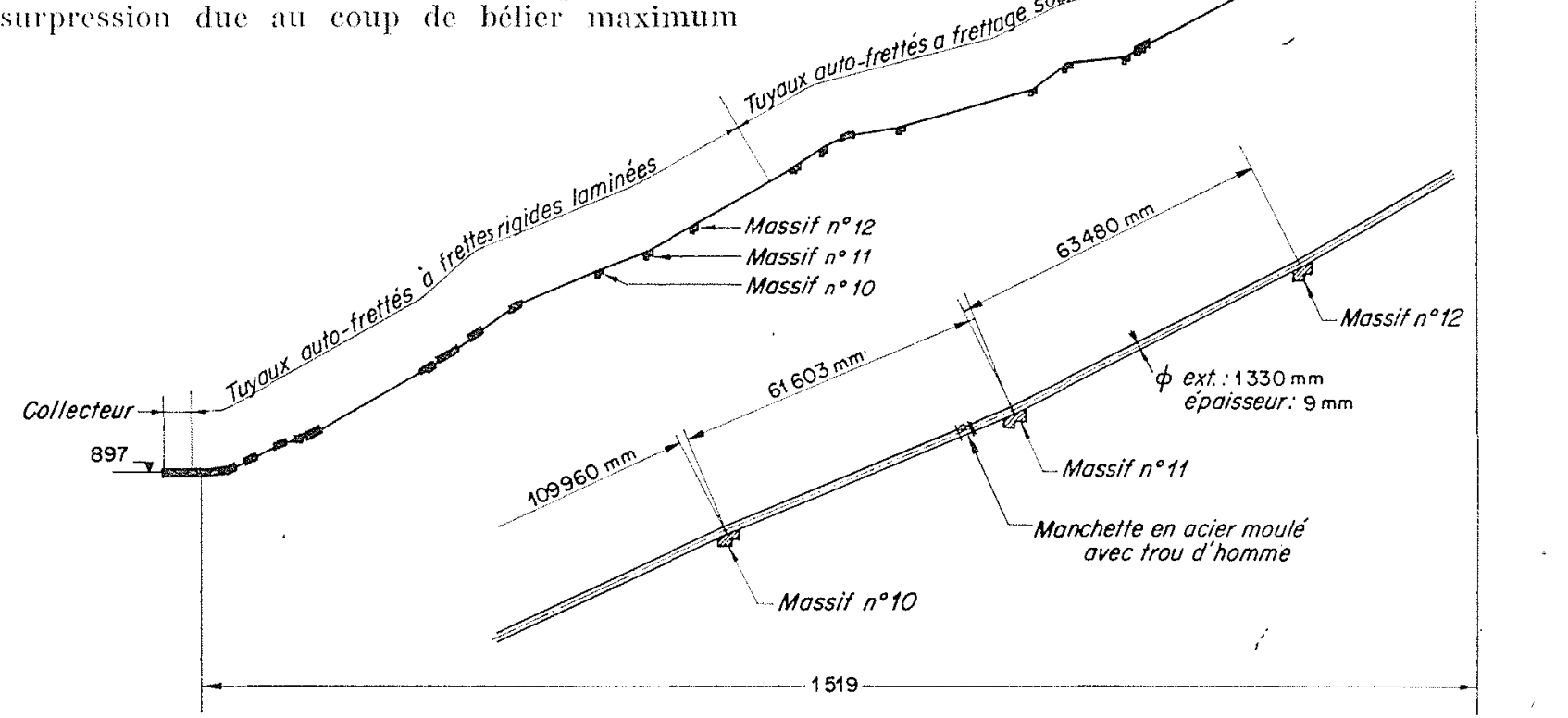

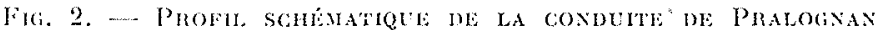

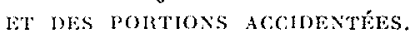




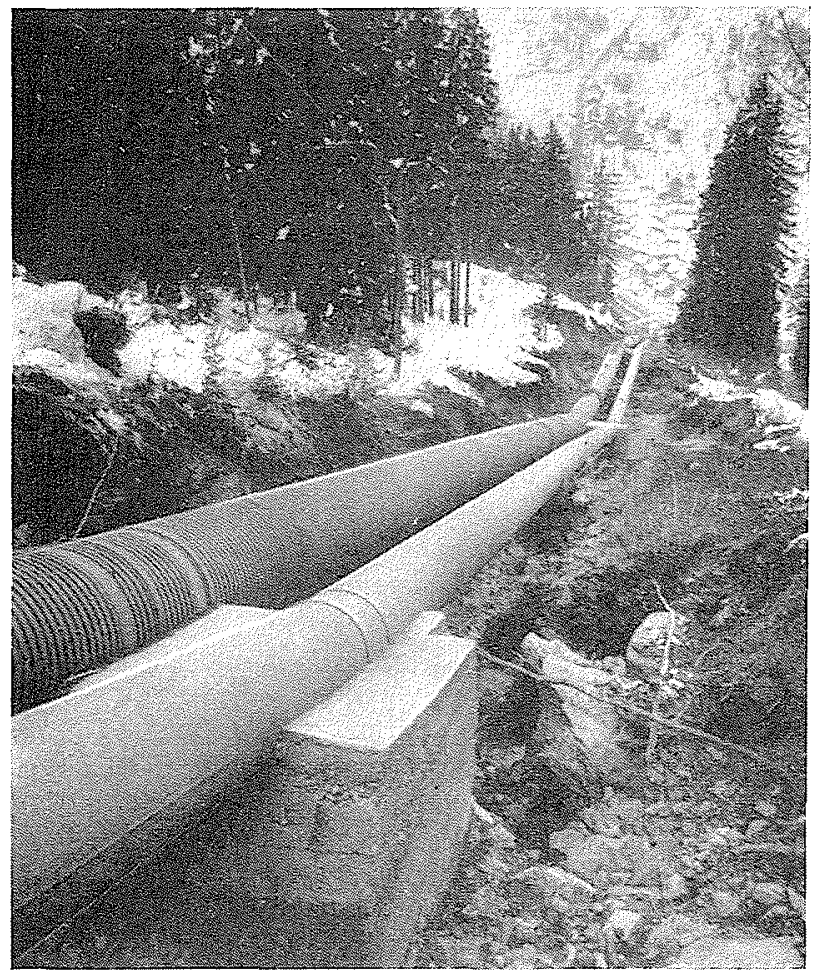

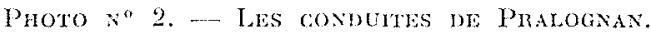

Massif $n^{\circ} 12$ a vue vers l'aval du ravinement.

(Cliche Fotellier-Moutiers.)

tes enterrées furent, sur $130 \mathrm{~m}$ environ, completement déchaussées et afrouillées entre les massif's d'ancrage $n^{\circ} 10$ à 12 , puis sur $50 \mathrm{~m}$ à l'aval du massif 10 (photos $3,4,5$ ).

Les conduites sont restées suspendues entri ces massifs, tous les piliers supports ayant éto emportés; la profondem du ravinement alleini 8 a $10 \mathrm{~m}$ entre les massif's 10-11 et 11-12.

Le massif n" 11 a quelque peu ralenti le flot et limité ainsi les déâts, mais ses fondations ont été arfouillées et il s'est abaissé de pris de $20 \mathrm{~cm}$.

Au moment de la rupture, la conduite forcós de la chute de Pralognan était en service. L'agent de service, s'etant rendu compte d'une anomalie, a provoqué l'arrèt des groupes, mais la conduite est restée en pression pendant deux heures environ, uniquement accrochée aux massif's d'ancrage nos 10 et 12 , le massif $n$ " 11 tirant par surerôt sur la conduite (photos nos 6 et 7 ).

Les cforts sur les deux conduites ont été considérables, du fait de l'afraissement du massif $n^{\circ} 11$ et de la flexion occasionnec par le poids de la conduite forcée en charge et de la conduite de décharge vide, les portées btant de $61 \mathrm{~m}$ entre les massifs 10 et 11 et $63 \mathrm{n}$ entre les massifs 11 et 12 .

Entre les massifs d'ancrage nos 10 et 11, il existe sur la conduite forcée une piece en acier coule portant un trou d'homme (visible sur la photo $n^{\circ}$ 8); cette pièce est assemblée aux tuyaux adjacents au moyen de brides et de tiges liletées qui portent aes traces tres nettes de deformations permanentes; celles-ci ont provoqué un écartement des brides of des fuites aux joints correspondants (joints plastiques). 'Cependant, aucune fuite n'ćtait constatée sur les soudures transversales, aussi bien pour celles faites au montage que pour celle faites en atelier, ce rui prouve la bonne qualité de leur exécution el leur exceptionnelle résistance.

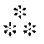

Pour remettre en service le plus rapidement possible la conduite foreée de Pralognan, on proceda a son relivement dams les zones atfaissces. Apres avoir desolidarise la conduite du massif $11 " 11$, on a installé des cries el des vérins Loul to long des parties à relever, ot on l'a ainsi remontéc de $25 \mathrm{~cm}$ an point le plus allaissé, entre les massifs 10 el 11. Lopération s'est efrectuće sans aucun dommage pour la conduite, qui a été calée à sa position définitive sur des calages provisoires conslitués par des chevalets en bois, qui seront remplacés par des supports définitifs des que les conditions climatériques

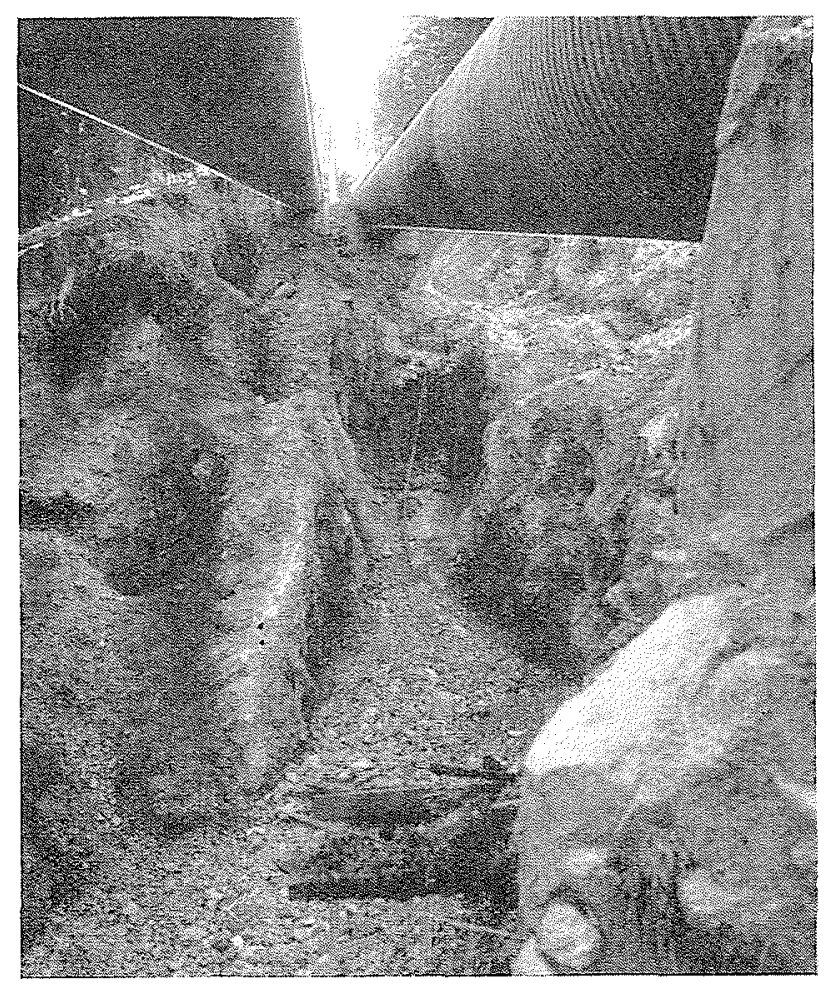

PHOTO N" 3. - VUE PRISE DU MASSIF N" 9 VERS L'AMON'. (Cliché Hotellier-Moutiers.) 
le permettront. Le massif d'ancrage $n^{\circ} 11$ a été remis en état par des reprises en sous-œuvre, par la réfection de la partie supérieure et par des scellements qui le lient à la conduite.

Les boulons de jonction de la manchette en acier coulé, cutre les massifs 10 et 11 , ont été changés, et la conduite a été remise en service sur ses calages provisoires le 24 décembre 1952.

Elle fonctionne depuis comme si rien de fâcheux ne lui était survenu.

$$
* *
$$

Avant 1910, on ne connaissait en France que les conduites rivces, qui donnèrent lieu jusqu'en 1907 à de graves mécomptes, puisque, pour certaines d'entre elles, des ruptures se produisaient dès la première mise en charge sous la pression statique seulement. A cette époque, en effet, les trous de rivets étaient simplement poinçonnes et cetle opération de poinconnage avait les plus désastreuses conséquenees sur la structure de l'acier utilisé. Dès que le poinconnage fut remplacé par un perçage suivi d'un alésage, tout rentra dans l'ordre et l'on n'eut plus jamais d'accident à constater en cours d'exploitation sur les conduites rivées, qui, du tait de leur structure, ne pouvaient cependant subir au préalable d'épreuve hydraulique síricuse (défaut

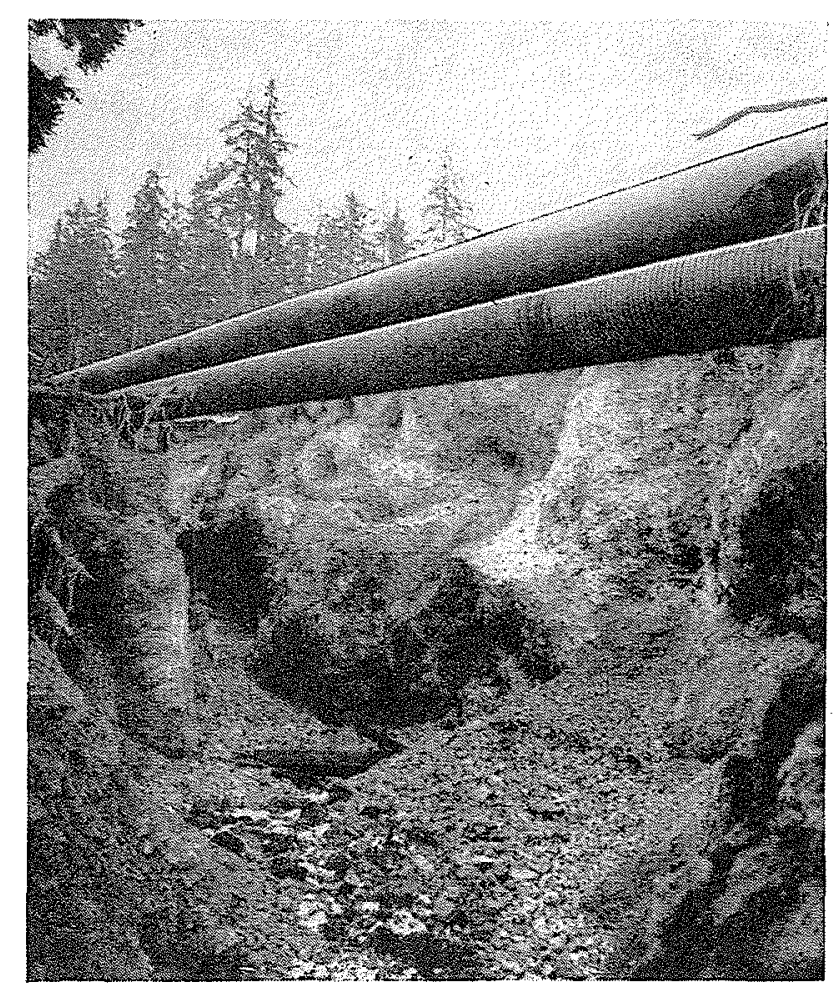

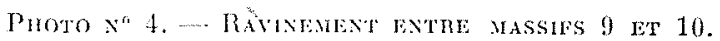
(Cliché Hotellier-Nonticrs.)

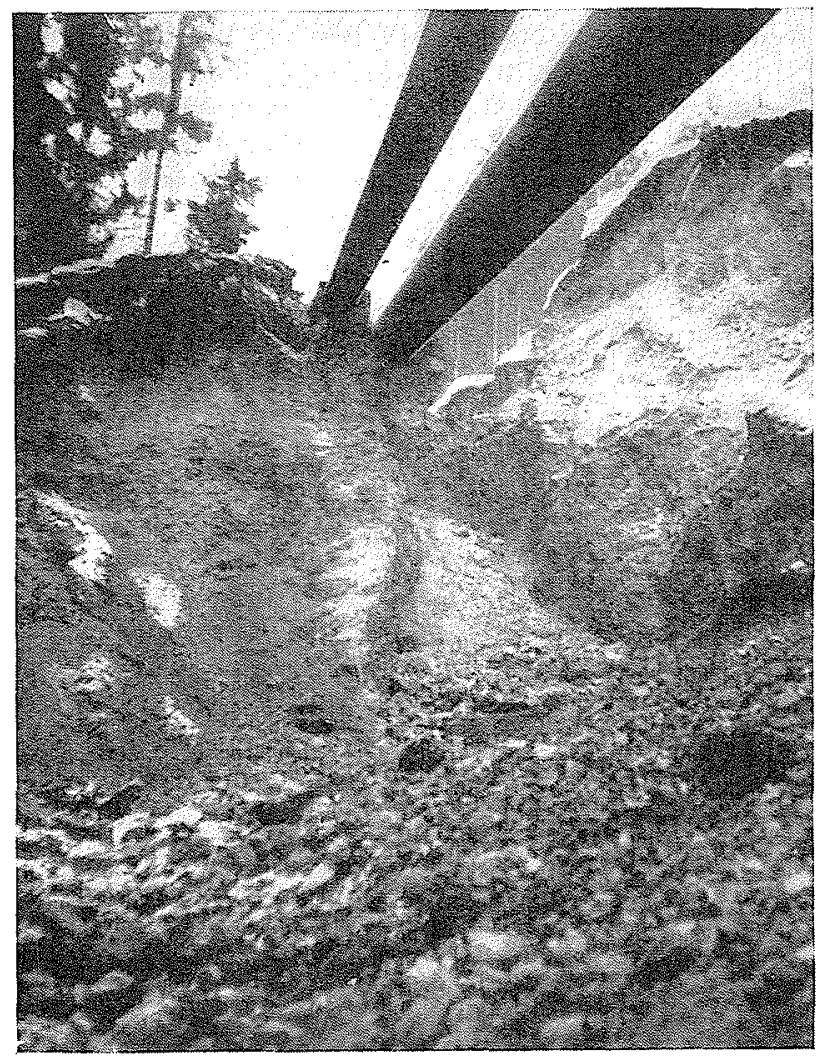

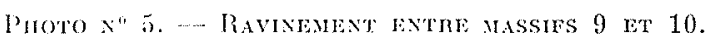

(Clichs S.D.E.M.)

d'ćtanchéité des rivures). D'autre part, on savait depuis longtemps que le cintrage à froid ne nuisait pas aux qualités de l'acier et l'on pouvait ainsi compter avec certitude sur la résistance d'une rivure.

En 1910, lorsque l'on commenca ì utiliser la soudure au gaz ì l'eau ì l'échelle industrielle et jusqu'au moment où on l'abandonna, vers 1930 , au profit de la soudure électrique, on n'avait aucun moyen d'investigation permettant de déceler les défauts et les modifications de structure du métal inhérents à l'opération de soudage, que les traitements thermiques, indispensables à la régénération du métal de la zone de soudure, ne faisaient pas toujours disparaitre et que larrondissage à chaud du tuyau déformé augmentaient bien souvent. La soudure au gaz at l'eau avait contre elle un préjugé de fragilité.

Un seul moyen de contrôle restait à la disposition des constructeurs : l'épreuve hydraulique cn usine.

A l'étranger, en Allemagne notamment, où l'on fabriquait bien avant 1910 des tuyaux soudés au gaz à l'eau, l'épreuve était faite à 1,5 fois la pression de service, prise bien souvent égale à la charge statique. C'est cette base que l'on prit comme point de départ en France. 
Malgré cette marge de $50 \%$, qui paraissait importante, de nombreuses ruptures de conduites soudées au gaz à l'eau étaient constatées aux essais de mise en service ou en cours d'exploilation. Le contróle à $1, \overline{5}$ s'avérant ainsi insuffim sant, dis 1918 on admit en France qu'une conduite forcée devait etre etablie en tenant compte :

- Des surpressions maximums résultant du fonctionnement normal des turbines ou des pompes qui recoivent ou refoulent l'eau en pression;

D'un coefficient de sécurité égal à 2,5 par rapport à la limite élasticue du métal qui la constitue;

De la vérification d'un coefficient de sécurité réel et effectif de 2 par une épreuve hydraulique en usines at double de la pression maximum nomale de marche (pression statique + surpression maximum due au fonctionnement normal des turbines on des pompes) des divers éléments de la conduite forcée considérée.

$$
*
$$

Nous avons eu bien souvent l'oecasion de donner les raisons qui nous ont conduils à adopter cette "règle d'or», que nous arons considérée jusqu'à présent comme la condition nécessaire ot suffisante à la bonne tenue d'une conduite forcée dans le temps de son exploitation.

Depuis lors, aucune rupture n'a été enregistrée sur des conduites dont les ćléments ont ainsi été éprourés. Est-ce à dire que de telles conduites sont à l'abri de tout aceident du genre de celui qui s'est produit sur la conduite du Doron de Champagny? Nous le pensons, car la pression résultant d'un coup de bélier accidentel et exceptionncl à transmission intégrale, qui peut toujours se produire dans l'existence d'une conduite (apres quarante ans pour Champagny), n'atteint en aucun point - d'apres les constatations que nous avons pu faire depuis près d'un demi-siècle -.. la pression d'épreuve en usine lorsque celle-ci est faite au double.

Cependant, les constatations faites sur la conduite de Champagny sont assez troublantes:

La rupture ne s'est pas produite sur la ligne des bords soudés, mais dans la partie extrême de la zone de soudure (celle qui a été chaufféc au blanc soudant et martelée, et qui peut atteindre plus de $15 \mathrm{~cm}$ pour les fortes épaisseurs).

Il n'y a ni décollement ni inclusion; la cassure est nette et sèche, e'est le type même de la rupture de fragilité.
Si une conduite lorcée était soumise à des efrorts alternés, on pourrait craindre que cette zone, fragile dès l'origine, ait subi dans le temps une transformation structurale avec augmentalion considérable de la fragilité. Mais ce n'est pas le cas d'une conduite forcée, qui ne subit que des efforts ondulés de faible amplitude; par ailleurs, les coups de bélier dus au fonctionnement normal des machines n'agissent pas comme un choc, vu le temps non négligeable de leur développement, et nous croyons fermement que la rupture constatée se serait produite en usine en 1912 si le tuyau correspondant avait été éprouvé au double et non à 1,5 , et cela mal-

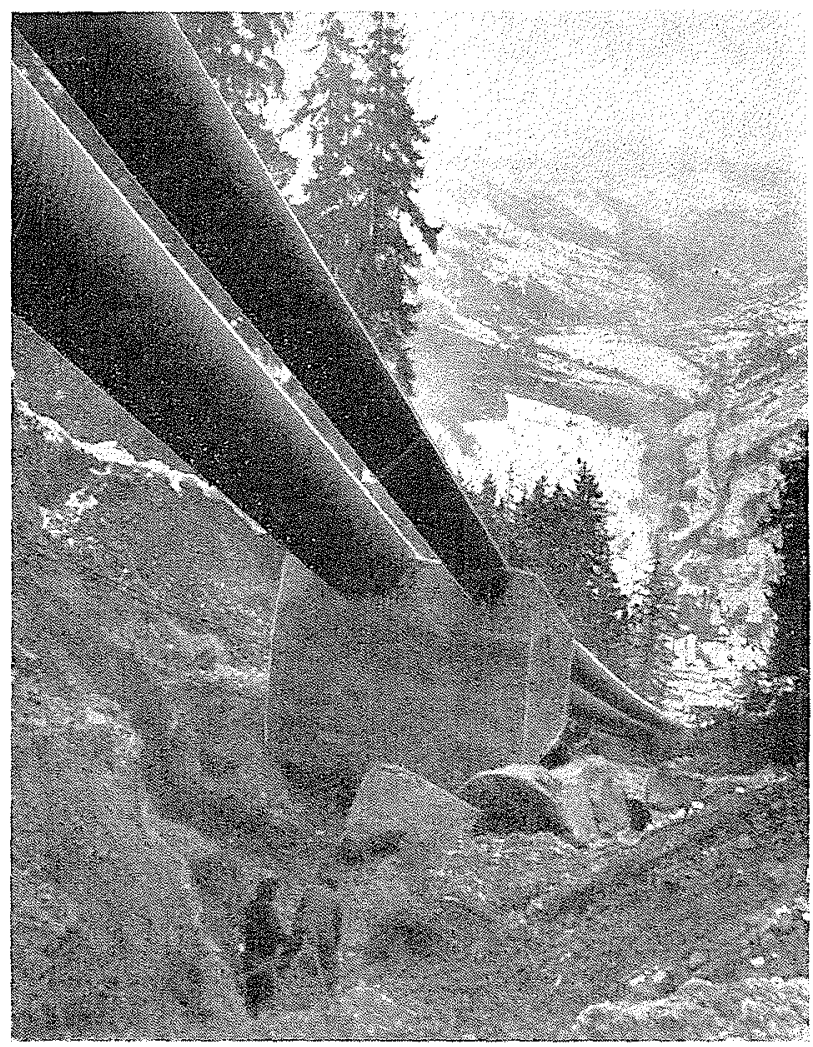

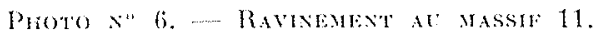

gré les résultals d'un essai de rupture fait sur la partie de ce tuyau restée intacte; car le degré le fragilité d'une soudure au gaz à l'eau est parfois très différent d'un point à un autre de la zone de soudure d'un même tuyau.

Cette rupture s'est en effet produite à une pression supérieure au double de la pression de service, alors que la limite ilastique venait seulement d'être atteinte, 'puisque la déformation permanente mesurée après les essais était de $0,16 \%$, inférieure à $0,2 \%$, correspondant à la limile ćlastique apparente. 
Depuis 1930, on a abandonné en France la soudure au gaz à l'eau au profit de la soudure électrique, avec laquelle on dispose de moyens d'investigation et de contrôle que l'on n'avait pas auparavant et qui permettent de déceler avec certitude, avant les épreuves hydrauliques, tous les défauts ou vices tels que inclusions, criques et fissures.

Depuis que nous construisons des tuyaux soudés à l'arc électrique, aucun incident ne s'est jamais produit; cependant, nous connaissons certaines conduites qui, du fait du coup de bélier accidentel et exceptionnel, ont été mises à rude épreuve. Il suffit de rappeler le cas de la conduite

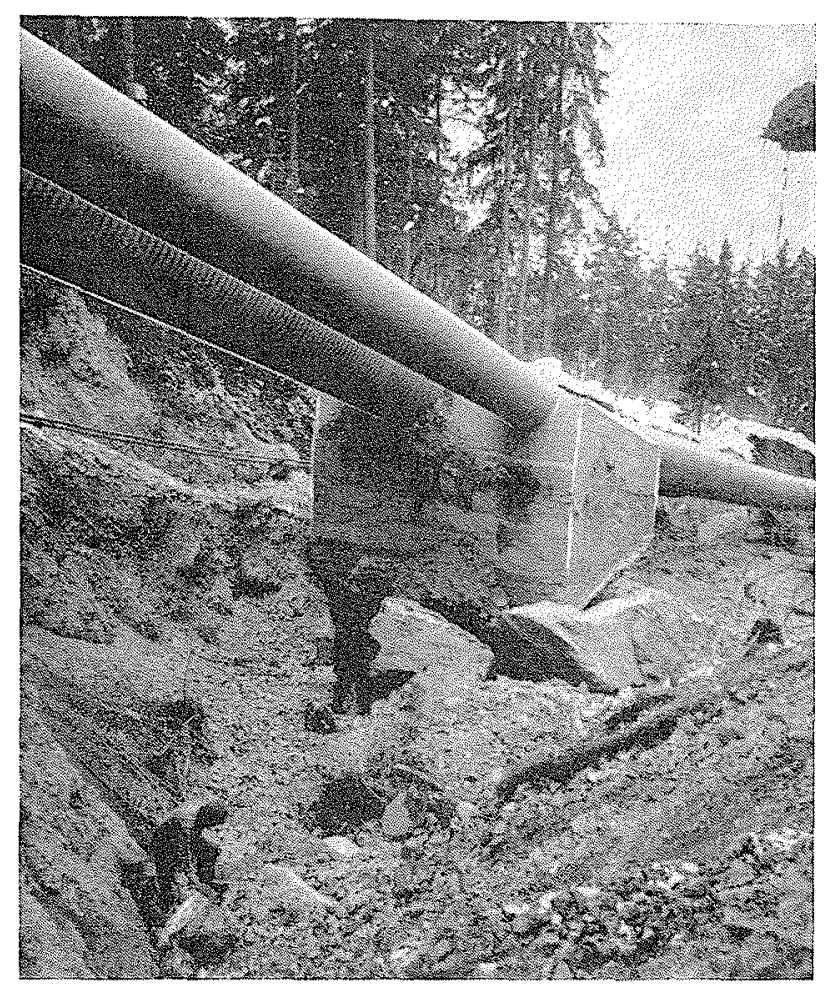

Photo $N^{\circ}$ 7. - Ravinementi aU Massef $11 \mathrm{VU}$ DE L'amont.

(Cliché Hotellier-Montiers.)

d'Izourt en 1938 (le coup de bélier accidentel était la conséquence du choc produit par une avalanche) et celui de la conduite de Pontcharra-sur-Breda en 1950 (le coup de bélier accidentel s'est produit au moment des essais de mise en service, à la suite d'une fermeture des distributeurs en ún temps inférieur à $2 \mathrm{~L} / a$ ).

\section{$* *$}

Les tuyaux soudés à l'arc électrique constituent le point de départ pour la fabrication des tuyaux auto-frettés et surpressés qui équipent actuellement les conduites forcées modernes de haute chute à grande puissance.

Ainsi qu'on le sait, lors de la fabrication des tuyaux auto-frettés et des tuyaux surpressés, la limite élastique du métal est largement dépassée.

Au cours de ces fabrications, que nous faisons depuis vingt ans déjà, nous avons été amenés à laire d'importantes constatations, qui sont les suivantes :

1. -.- Lors des opérations d'auto-frettage et de surpressage, aucune rupture de virole n'a jamais été enregistrée avant que la limite élastique du métal soit atteinte. (Pour un acier au $\mathrm{Cr}-\mathrm{Cu}$ à $54 \mathrm{~kg} / \mathrm{mm}^{2}$, limite élastique $34 \mathrm{~kg}$, l'épreuve au double du tuyau soudé correspondant donnerait une fatigue de $27 \mathrm{~kg}$, pour un coefficient de sécurité théorique de 2,5 , et $34 \mathrm{~kg}$ pour un coefficient de sécurité théorique de 2 , qui se trouverait ainsi vérifié expérimentalement).

2. - Au cours de ces mêmes opérations, des ruptures de viroles, très rares il est vrai, se sont produites alors que la limite élastique avait été dépassée, ruptures sur des soudures que le contrôle radiographique avait révélées impeccables. Les viroles correspondantes avaient subi les traitements thermiques habituels avant et après soudure et les tôles qui les constituaient avaient subi chez le fournisseur. les essais de réception les plus complets. Par ailleurs, elles avaient subi un recuit de normalisation et des essais de soudabilité concluants.

Les examens, contrôles et essais ultérieurs ont prouvé qu'il s'agissait de ruptures de fragilité dont le point de départ se situait en des zones réduites et localisées devenues fragiles après soudure. L'éxécution de la soudure n'avait rien révélé de particulier, mais des modifications de structure impossibles à déceler autrement que par une épreuve hydraulique poussée jusqu'au dépassement de la limite élastique du métal s'étaient produites au soudage. (L'essai du tuyau de la conduite de Champagny dont il est fait mention plus haut confirme ce point de vue.)

3. - La limite élastique dépassée, aucune rupture prématurée ne s'est jamais produite, même pour des déformations plastiques importantes.

Lors de notre communication au $4^{\circ}$ Congrès 
International des Fabrications Mécaniques, à Stockholm (juin 1952)* : "Utilisation du principe du relevement de la limite élastique des aciers par écrouissage et vieillissement artificiel à la construction des conduites forcées et économie en résultant $»$, nous avions tiré un certain nombre de conclusions, confirmées par ce qui précède :

On peut tout d'abord affirmer que l'emploi des tuyaux surpressés et des tuyaux auto-frettés surpressés dome à l'exploitant la sécurité la plus complite qu'il soit possible d'obtenir en matière de conduites forcées. Le coefficient de sécurité est vérifié expérimentalement par rapport à la limite élastique réelle du métal utilisé, qui, on le sait, est toujours supérieure au minimum théorique, c'est-à-dire que le coefficient de sécurité réel est toujours supérieur au coefficient de sécurité théorique.

On peut également affirmer que pour les tuyaux surpressés et les tuyaux auto-frettés surpressés, il n'y a aucun inconvénient à réduire le coefficient de sécurité théorique admis jusqu'à maintenant jusqu'au minimum compatible avec l'épreuve au double, c'est-à-dire : 2.

Pour les tuyaux simplement soudés, une sécurité du même genre ne peut être obtenue qu'autant que l'épreuve hydraulique est poussée jusqu'à atteindre une déformation plastique de 0,5 i $1 \%$ en construisant, ainsi que nous avons déjà en l'oceasion de le faire pour une importante conduite forcée en Italie, des tuyaux à « limite élastique ef coefficient de sécurité contrôlés ».

Cela permet d'envisager pour les tuyaux soudés la réduction jusqu'à '2 du coefficient de sécurité, comme pour les tuyaux précédents, el de réaliser ainsi de nouvelles el importantes économies de métal.

\section{*}

En ce qui concerne les conduites rivées poinçonnées et toutes les conduites soudées au ga\% à l'eau éprouvées à moins de 2 fois la pression. de service, d'autres conclusions peuvent encore. être tirées :

Pour les premières, il faudrait admettre de les remplacer ou de les renforcer dans la proportion de 50 à $100 \%$. Pour les secondes, on devrait, à notre avis, et selon leur état, procéder à leur renforcenient dans la proportion de 30 à $50 \%$. Ces renforcements sont d'ailleurs faciles à réaliser et peuvent se faire sur une conduite en service par le procédé que nous avons déja

\footnotetext{
* Cette communication fera l'objet d'une publication dans un très prochain numéro de la Houlle Blanche.
}

utilisé, en particulier sur la conduite du Doron de Champagny elle-même, dans sa traversée de la nouvelle centrale de Pralognan. (Voir l'article de M. Beaumel: « La conduite forcée de la chute de Pralognan. »La Houille Blanche, numéro de mars-aviil 1950.)

Ces renforcements effectués, nous croyous indispensable de faire un essai de vérification, à la pression hydraulique, de l'ensemble de la conduite par la méthode utilisée maintenant sur toutes les conduites forcées modernes, dite « d'essai après montage à surpression uniforme ». Cette méthode consiste à introduire au sommet de la conduite une surpression au-des-

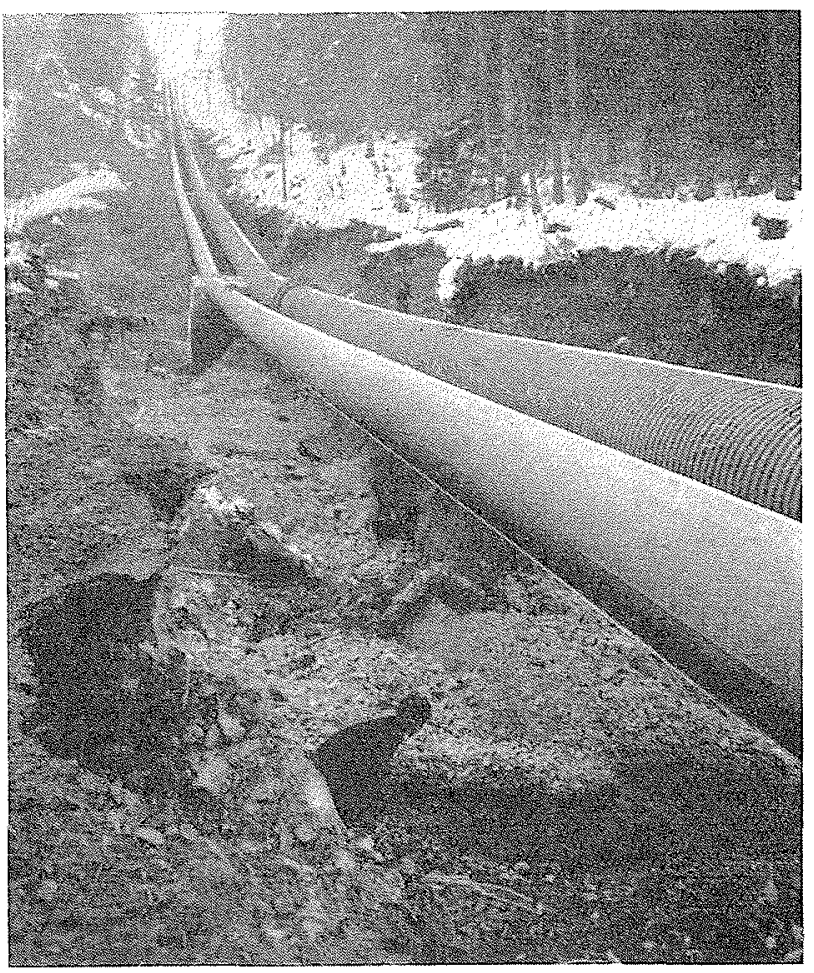

Photo N $^{\circ}$ 8, - Vue prise du Massif 10 vers l'amont. (Cliché Hotellier-Moûtiers,)

sus de la charge statique, dont la valeur correspond sensiblement à celle du coup de bélier exceptionnel accidentel envisagé dans chaque cas particulier et limitée à $100 \%$ de la pression maximum normale de marche à la partie inférieure de la portion de conduite comportant la plus faible épaisseur.

$$
\text { **: }
$$

L'accident survenu à la conduite du Doron de Champagny nous amène à rappeler aux maitres de l'œuvre la sécurité que présente, pour l'exploitation des conduites forcées, la méthode 
dite "française », c'est-à-dire celle qui ne comporte aucun joint de dilatation. On frémit, en effet, à la pensée de la catastrophe qui se serait produite du fait du déboîtement inévitable des joints de dilatation si la conduite de Pralognan en avait été munie.

La méthode «française » a recu la sanction de l'expérience de plus d'un demi-siècle, elle est appliquée à toutes les conduites forcées moder. nes que nous avons installées, tant en France qu'à l'étranger, et en particulier aux conduites de hautes chutes à grande puissance, où tous les coudes sont ancrés.

Lorsque la conduite d'Izourt, composée exclusivement de tuyaux soudés, s'est trouvée sus- pendue sur une grande longueur, après que ses supports eussent été emportés par une avalanche, et qu'elle eut fonctionné pendant plusieurs heures dans cette position critique sans aucun dommage, on pouvait se demander ce qui se serait passé si elle avait été constituée de tuyaux auto-frettés, dont l'épaisseur peut atteindre, ainsi qu'on le sait, le cinquième de l'épaisseur normale des tuyaux soudés.

L'accident que nous venons de relater en détail a permis de constater la remarquable tenue des tuyaux auto-frettés, mểme dans les cas les plus imprévus, et cela est aussi de nature à donner tous apaisements aux Exploitants.

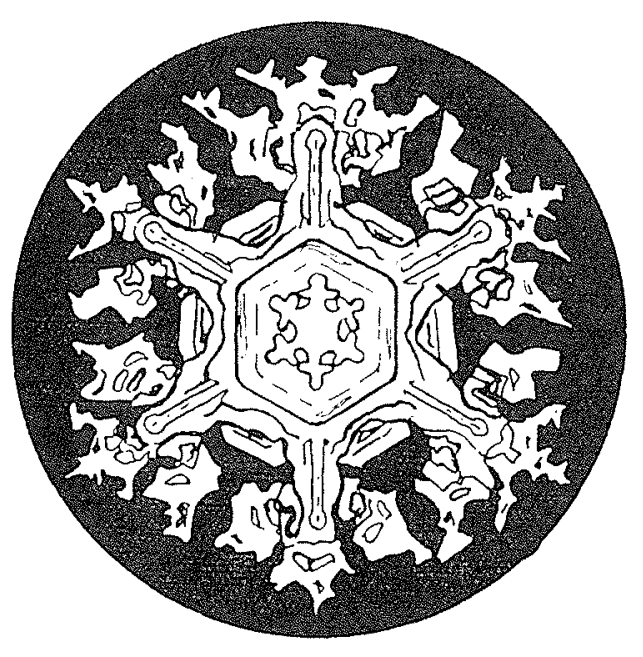

